\title{
A Generic Handover Decision MANAGEMENT FRAMEWORK FOR NEXT GENERATION NETWORKS
}

\author{
Shanthy Menezes ${ }^{1}$ and S. Venkatesan ${ }^{2}$ \\ ${ }^{1}$ Department of Computer Science, University of Texas at Dallas, Richardson, TX, USA \\ ${ }^{1}$ shanthy.menezesestudent.utdallas.edu \\ ${ }^{2}$ venky@utdallas.edu
}

\begin{abstract}
Next generation networks are defined to be packet based networks that provide telecommunication services to users by utilizing different transport technologies, wired and wireless. In this paper we provide a generic framework for handover decision management in next generation networks. We show that any handover decision algorithm can utilize our framework. We show the feasibility of applying our framework through an implementation example. One of the main features of NGNs is application adaptability; the applications designed for NGN systems have to be adaptable to different networks and different devices. We propose a handover decision algorithm that utilizes our generic framework and selects the network for handover such that the quality of experience of the user is near optimal. Through simulations we show that our algorithm reduces unnecessary handovers and also satisfies the handover requirements.
\end{abstract}

\section{KEYWORDS}

Next Generation Network, Wireless Network, Mobility, Handover Decision

\section{INTRODUCTION}

Next generation networks (NGNs) are expected to support a variety of the existing wired/wireless access network technologies as well as new wireless access technologies to support high data rates up to $1 \mathrm{Gbps}$. The network environment envisioned for NGN is made up of the various wired and wireless access networks connected to a common NGN core network. Figure 1 shows a typical setting of a NGN.

Since the evolution towards NGNs is driven by the ever increasing mobile subscriber base and the set of mobile applications, mobility management (MM) is an essential requirement for NGN users. Hence the Telecommunication Standardization Sector (ITU-T) has described fifteen general mobility management requirements for NGNs [1]. One of the requirements is to support policy-based and dynamic network selection. Network selection is the process of choosing the access network to connect to. The network selection process is done initially at the time of starting a service (application) that requires network connection and during handover of the service to a new network. During handover, the selection process is known as the handover decision process; that is, deciding whether to disconnect from the current network and deciding which network to handover to.

During handover in NGN, the mobile device has the option of handing over to the access network which is of the same radio access technology (RAT) as its current network (horizontal handover) or to an access network of a different RAT (vertical handover). Traditionally a handover is performed when the current network does not meet the requirements of the mobile device. In the case of NGNs the handover process poses a variety of challenges due to the different set of applications used, networks available, quality of service (QoS) available, and devices used. In this environment handover can be performed not only because the current 
network does not meet the requirements, but also due to the availability of a better network or device.

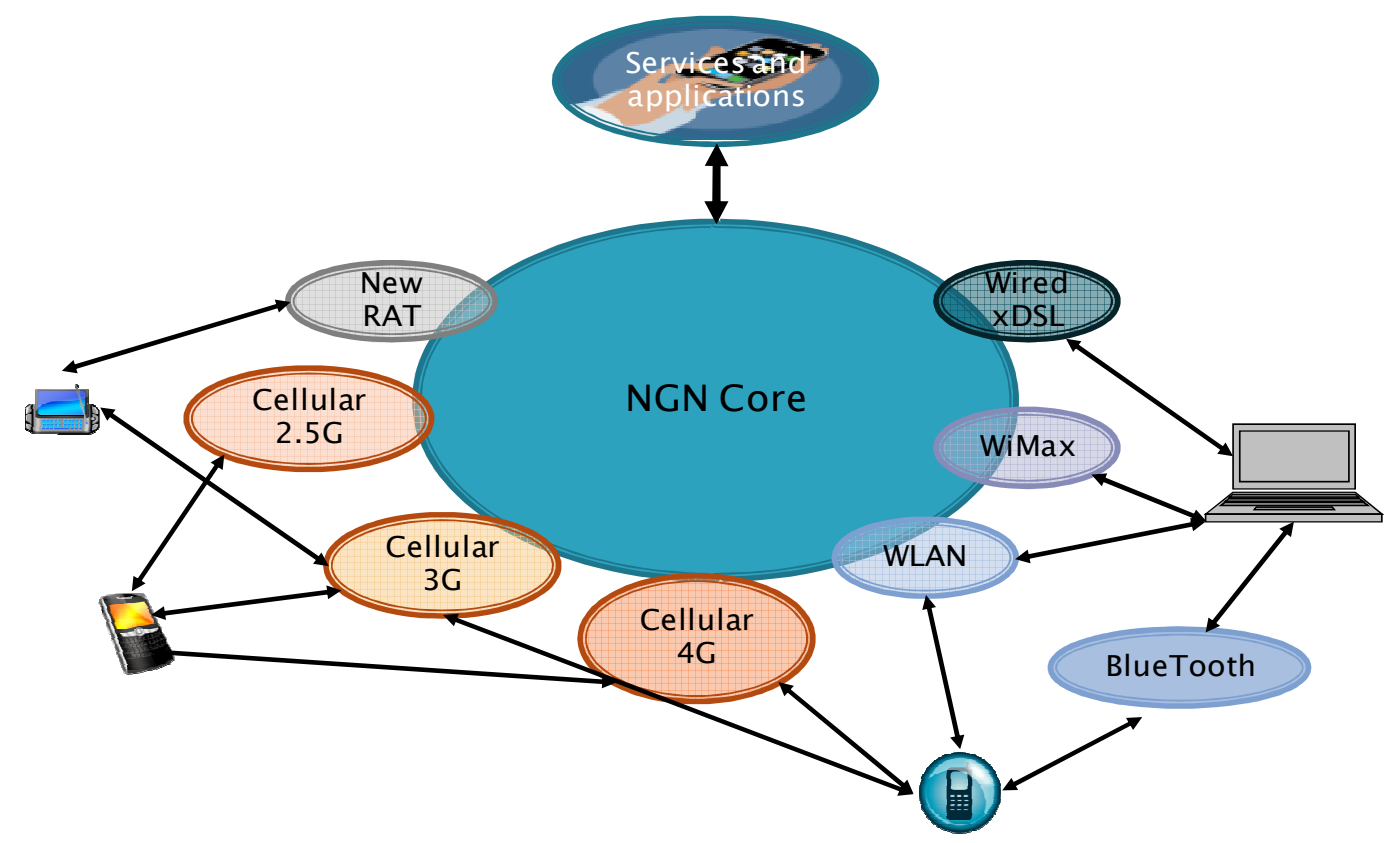

Figure 1. Architecture of Next Generation Networks

The requirements of horizontal handovers were primarily confined to the radio link requirements such as radio signal strength and bit error rate of the channel. In the NGN scenario, the variety of access networks available differ in QoS provision, security schemes offered, cost of service etc. The capabilities of the mobile devices also vary in many aspects such as power consumption, application support and so forth. The key goal for NGNs is to enhance the user experience. This requires the provision of the most suitable radio access network for any given user. Thus the handover decision has to be managed such that the requirements of the user are satisfied optimally.

In this paper we propose a generic framework for handover decision management which has an extensive list of requirements for handover that optimize the mobile user experience. We elaborate on actual implementation of our framework by utilizing the IEEE 802.21 media independent handover standard. In NGNs, in order to be able to offer services anywhere and anytime the services and applications have to be designed such that they are adaptable to the multitude of access networks available. We propose a handover decision algorithm that takes into consideration the needs of adaptable applications and also provides flexibility in specifying the user and network requirements. Through simulations we show that our algorithm satisfies user requirements and also reduces unnecessary handovers.

The rest of the paper is organized as follows. Section 2 discusses related work in the area of handover decision algorithms and framework suitable for NGNs. In section3 we present our generic framework for handover decision management. In section 4 we show how our framework can be used to represent any existing or future handover decision algorithm through three examples consisting of a decision algorithm for horizontal handover and two of the most cited decision algorithms for vertical handover. Section 5 presents our proposed generic handover decision algorithm. In section 6 we present an example implementation of our generic framework and handover decision algorithm. Section 7 provides the simulation details and analyzes the simulation results. Finally section 8 provides the summary of the paper. 
International Journal of Computer Networks \& Communications (IJCNC) Vol.3, No.2, March 2011

\section{RELATED WORK}

Various solutions $[2,3,5,7,8,9,12,13,14,15]$ for handover management in heterogeneous network environment have been proposed in the literature. Several solutions $[2,3,14,15]$ utilize the services provided by the IEEE standard 802.21 [4] to facilitate a handover decision. The 802.21 framework can provide the parameters required for a handover decision; an actual handover decision management module is not specified in the standard. Cacace and Vollero [2] provide a detailed mobility management framework that uses the 802.21 standard to provide services to the applications to adapt to the new networks available. Their solution provides input to the mobile IPv6 protocol to implement a handover without any packet loss. The mobility management frameworks provided in $[2,3,5]$ do not address the handover decision that decides the link layer handover.

Yungkyu and Choi [6] provide solutions for handover between 802.16 and 802.11 networks that maximize the energy usage and minimize the cost required. These solutions are not universally applicable since not all the objectives for heterogeneous handovers are considered. A variety of approaches $[7,8,9]$ based on the theory of multi-attribute decision making (MADM) are generic in nature. We elaborate on these algorithms in section. 4 and also compare them to our handover decision algorithm using simulations. An extensive framework for handover decision management is not provided in these solutions $[7,8,9]$.

Any of the existing handover decision algorithms can be represented by our proposed framework. In section 4 we give examples of a horizontal handover decision algorithm and the two most cited vertical handover decision algorithms [7,9], and demonstrate how any handover decision algorithm can be represented using our generic framework. Due to the vast number of choices for access networks, a large discrepancy between the values offered by different networks is a definite possibility. The handover decision algorithm presented here addresses this issue with the aid of the framework provided. Our algorithm also prevents unnecessary handovers, unlike the other generic vertical handover algorithms.

\section{HANDOVER DECISION MANAGEMENT FRAMEWORK}

We present a handover decision management framework as shown in Figure 2. Any handover decision algorithm, whether for horizontal handover or vertical can be built upon our generic management framework. The vital part of our framework is a handover decision algorithm. The other parts of the framework are elements that affect the handover decision, used as inputs by the handover decision algorithm. The most influential of these inputs are the Parameters. Parameter values are influenced by the handover decision, namely the access network chosen. The parameters portray the objectives set by various entities that get affected by handover. The entities specify the requirements for the parameters in terms of weights and thresholds. The weights specify the importance of the parameters to the entity. The thresholds specify the conditions to be met for the parameters. The existing handover decision algorithms only consider one type of threshold that affects the handover decision, which we term mandatory threshold. In order to consider the adaptive nature of the services offered by NGNs, we consider another threshold for each parameter called the preferred threshold. The other feature of the framework is the values offered by the various networks for each of the parameters specified. The basis of any handover decision algorithm is a comparison of these offered values to the parameter requirements. Also as part of the framework, are the parameters used by handover decision algorithms to avoid unnecessary handovers.

In the next section we show that our framework can be used by any existing handover decision algorithm and we illustrate the utility through examples. If, due to future enhancements or restrictions to the NGNs the handover decision module requires inputs not represented by our framework, our framework can be expanded to include these inputs termed as future inputs. 




Figure 2. Handover Decision Management Framework

Our framework can be represented in vector form as follows:

\subsection{Entities}

The entities that provide the parameters are denoted by the vector $\left\langle\mathrm{E}_{1}, \ldots, \mathrm{E}_{\mathrm{M}}\right\rangle$.

\subsection{Parameter Requirements}

The parameters are denoted by $\left.<\mathrm{O}_{1}, \mathrm{O}_{2}, \ldots, \mathrm{O}_{\mathrm{P}}\right\rangle$. Parameter requirements are given in terms of weights and thresholds.

Weights: The weights specify the priorities given to each parameter. The higher the weight of the parameter, the larger is its influence in the handover decision. Each entity provides weights for its own parameters by prioritizing them, these weights are called local weights. The handover decision algorithm can prioritize the entities by providing weights to the entities. The global weights used by the algorithms are the products of the local weights and the weights of the respective entities. For example let $\left\langle\mathrm{W}_{\mathrm{i} 1}, \ldots, \mathrm{W}_{\mathrm{im}}\right\rangle$ be the local weights of the $\mathrm{m}$ parameters set by entity $i$ and $\left\langle\mathrm{W}_{\mathrm{j} 1}, \ldots, \mathrm{W}_{\mathrm{jn}}\right\rangle$ be the local weights of the $n$ parameters set by entity $j$. If the weights of $i$ and $j$ are $\mathrm{W} i$ and $\mathrm{Wj}$ respectively, then the global weights are $<\mathrm{W}_{\mathrm{i} 1} * \mathrm{~W}_{\mathrm{i}}, \ldots, \mathrm{W}_{\mathrm{im}} * \mathrm{~W}_{\mathrm{i}}, \mathrm{W}_{\mathrm{j} 1} * \mathrm{~W}_{\mathrm{j}}, \ldots, \mathrm{W}_{\mathrm{jn}} * \mathrm{~W}_{\mathrm{j}}>$. We denote the global weights of the parameter as $\mathrm{W}$ $=<\mathrm{W}_{1}, \mathrm{~W}_{2}, \ldots, \mathrm{W}_{\mathrm{P}}>$

Thresholds: In most of the handover decision algorithms in literature [7,8,9], the threshold values of the parameters are only considered in eliminating networks during the decision process. That is, if the network's offered value for a parameter does not meet a threshold, then it is not considered for handover. Since some of the requirements such as user requirements and service requirements are adaptable, a single threshold is not applicable in the case of handover in NGN networks. We propose the use of two types of threshold values:

1. Mandatory Thresholds: For a network to be eligible for selection, the network's offered value for a particular parameter should satisfy these thresholds. The mandatory thresholds are represented by the vector $\mu=\left\langle\mu_{1}, \mu_{2}, \ldots, \mu_{\mathrm{P}}>\right.$. These values could be lower sided, upper sided or exact depending on whether the value of the parameter should be $\geq, \leq$ or equal to the threshold. 
2. Preferred Thresholds: The preferred thresholds represent the values desired by users and services for their best performance. The preferred thresholds are either lower sided or upper sided. These may not be specified for all parameters. These are represented as vector $\pi=<\pi_{1}$, $\pi_{2}, \ldots, \pi_{\mathrm{P}}>$

\subsection{Parameter Values}

The values offered for each of the parameters by the access networks specifies the contribution of the network for each of these parameters. The values offered by an access network $\mathrm{i}$ is denoted as the vector $\mathrm{R}_{\mathrm{i}}=\left\langle\mathrm{R}_{\mathrm{i} 1}, \mathrm{R}_{\mathrm{i} 2}, \ldots, \mathrm{R}_{\mathrm{iP}}\right\rangle$. From the parameter values offered by the networks, we form two sets of vectors: (1) highest offered value $\mathrm{H}=\left\langle\mathrm{H}_{1}, \mathrm{H}_{2}, \ldots, \mathrm{H}_{\mathrm{P}}\right\rangle$ denoting the best offered value, and (2) lowest offered value $\mathrm{L}=\left\langle\mathrm{L}_{1}, \mathrm{~L}_{2}, \ldots, \mathrm{L}_{\mathrm{P}}\right\rangle$ denoting the worst offered. If parameter $\mathrm{O}_{\mathrm{j}}$ has a lower sided threshold, then $H_{j}=\max _{i} R_{i j}$ and $L_{j}=\min _{i} R_{i j}$. If $\mathrm{O}_{\mathrm{j}}$ has an upper sided threshold then $H_{j}=\min _{i} R_{i j}$ and $L_{j}=\max _{i} R_{i j}$, otherwise $\mathrm{H}_{\mathrm{j}}=\mathrm{L}_{\mathrm{j}}=\mathrm{R}_{\mathrm{ij}}$.

\subsection{System Parameters for Unnecessary Handover Avoidance}

If the handover decision is not implemented efficiently, it can result in unnecessary handovers that can also lead to the ping-pong effect. Hysterisis value and dwell timers are two such parameters utilized in horizontal handover decision algorithms. We represent the set of these system parameters by $\Sigma$.

\section{UTILITY OF THE FRAMEWORK}

Most handover decision algorithms can be divided into two phases (i) part of the algorithm that makes the decision to perform a handover and (ii) part of the algorithm that chooses the network to handover to. The first phase is referred to as the handover initiation algorithm and the second phases is referred to as the network selection algorithm. In this section we list some of the existing handover decision algorithms and show how they can be represented as functions of our framework.

Horizontal handover algorithm: One of the initial handover decision algorithms used for horizontal handover part of The North American Personal Access Communication Systems (PACS) combines a hysteresis value with dwell timer to reduce the number of unnecessary handovers [10]. This basic algorithm can be split into initiation algorithm and network selection algorithm.

Initiation algorithm: If $R_{c 1} \leq \mu_{1}$ and $R_{c 2} \leq \mu_{2}$ for $\Delta T$ amount of time then handover to the network selected by the network selection algorithm. The initiation algorithm can be represented as $\mathrm{f}\left(R_{c l}\right.$, $R_{c 2}, \Delta T, \mu_{1}, \mu_{2}$ ), where c represents the current network, $R_{c l}$ is the RSS measured and $R_{c 2}$ is the bit error rate measured.

Network selection: For the network selection, the mandatory thresholds are computed as $\mu_{1}=$ $R_{c 1}+\delta_{1}$ and $\mu_{2}=R_{c 2}+\delta_{2}, \delta_{1}$ and $\delta_{2}$ are hysteresis values used to avoid unnecessary handover. If $R_{i 1} \geq \mu_{1}$ and $R_{i 2} \geq \mu_{2}$, for any detected neighboring network $i$, then handover to network $i$. The network selection algorithm can be represented as $f\left(R, \mu_{1}, \mu_{2}, \Sigma\right)$

Simple additive weighing (SAW) algorithm: Various vertical handover algorithms are proposed in literature based on SAW [8]. SAW is a network selection algorithm. According to SAW the selected network is the one satisfying $\max _{i \in M} \sum_{j=1}^{P} W_{j} R_{i j}$, where $\mathrm{M}$ is the set of all eligible networks, $W_{j}$ is weight of parameter $j$, and $R_{i j}$ is the value offered by network $i$ for parameter $\mathrm{j}$. This algorithm can be represented as $f(W, R)$. 
Multiservice vertical handover: A variation of SAW proposed by Zhu and McNair [7] is to use the cost function for each network i give by: $C_{i}=\sum_{s} C_{i}^{s}$, where $C_{i}^{s}$ is the cost incurred by choosing network $i$ for service $s$ and is given as $C_{i}^{s}=\sum_{j} W_{j} \eta\left(R_{i j}\right)$. The function $\eta$ is used to normalize the values offered. Only those $\mathrm{R}_{\mathrm{ij}}$ values that satisfy the mandatory thresholds $\mu$ are considered. Whenever a new network is detected, the cost function is evaluated for all networks and the network with the minimum cost is selected. Hence the handover selection is done whenever a new low cost network is detected.

The authors also present a per service handover algorithm. The services are prioritized, and each service in the order of the priorities is handed off to the network with the least cost. The assumption here is that the mobile device is communicating using multiple radio interfaces simultaneously. This algorithm can be represented as $f(\mu, W, R)$.

Gray relational analysis (GRA) algorithm: The GRA algorithm presented by Song and Jamalipour [9] listed in Figure 3 is based on grey relational analysis (GRA). GRA is used to analyze relationship between different discrete vectors. A grey relational coefficient (GRC) is computed between each of the vectors and a reference vector known as the ideal vector. The vector with the largest GRC is selected.

\section{GRA Algorithm $(\mu, W, R, H, L)$}

$1 \quad$ Normalize the vectors $\mathrm{Ri}$ into a vector $R_{i}^{*}=\left(R_{i 1}^{*}, \ldots, R_{i P}^{*}\right)$

2 Calculate the GRC for each network as $G R C_{i}=\frac{1}{\sum_{j=1}^{P} W_{j}\left|R_{i j}^{*}-1\right|+1}$

3 Select the network i with minimum GRC

Figure 3 GRA Algorithm

Here $R_{i j}^{*}=\frac{R_{i j}-L_{j}}{H_{j}-L_{j}}$ if parameter $\mathrm{O}_{\mathrm{j}}$ has a lower sided threshold and $R_{i j}^{*}=\frac{L_{j}-R_{i j}}{L_{j}-H_{j}}$. This network selection algorithm can be represented as $f(\mu, W, R, H, L)$.

\section{Generic Handover Decision Algorithm}

Clearkt no decision algorithm considers the preferred threshold values of adaptable parameter requirements. In this section we describe a handover initiation and network selection algorithm that can optimize the user quality of experience by considering the requirements for the best operation of the application/service used.

\subsection{Definitions}

For a network $\mathrm{i}$, and a parameter $\mathrm{j}$, we quantify digression from preferred threshold as $\Delta D_{i j}$ :

$\Delta D_{i j}=0$ if preferred threshold is satisfied, i.e if $R_{i j} \geq \pi_{j}$ or $R_{i j} \leq \pi_{j}$ depending on whether the threshold is lower sided or upper sided respectively.

If the preferred threshold is not satisfied then the digression is given as:

$\Delta D_{i j}=\left(\pi_{j}-R_{i j}\right) / \pi_{j}$, if threshold is lower sided.

$\Delta D_{i j}=\left(R_{i j}-\pi_{j}\right) / \pi_{j}$, if threshold is upper sided. 
The digression for a network $i$ is given by $\Delta D_{i}=\sum_{j=1}^{P} \Delta D_{i j}$.

\subsection{Algorithm}

Our handover decision algorithm consists of two phases, as described in Figure 4.



Figure 4 Handover Decision Algorithm

\subsubsection{Phase I: Handover Initiation Algorithm}

Handover is initiated if one of the two conditions is met:

Condition 1: The current network has not met the threshold requirements for $\Delta \mathrm{T}$. That is: there exists at least one parameter $j(1 \leq j \leq P)$, such that $R_{c j}<\pi_{j}$ or $R_{c j}>\pi_{j}$ for lower sided or upper sided mandatory threshold respectively for at least $\Delta \mathrm{T}$ amount of time.

Condition 2: Condition 1 is not true, but one or more new networks are detected and for at least one of the new networks $i, \Delta D_{i}<\Delta D_{c}$, where $\Delta D_{c}$ is the newly calculated digression for the current network.

\subsubsection{Phase II: Network Selection Algorithm}

Calculate $\Delta D_{i}$ for each of the detected networks and select the network with the minimum $\Delta D_{i}$ value. If handover was initiated due to condition 2 in phase I, then handover to the network with minimum $\Delta D_{i}$ value only if the digression for current network $\Delta D_{c}<\Delta D_{i}$.

We also propose a variation of the handover decision algorithm described above, which incorporate the weights of parameters in calculating $\Delta D_{i}$. Each parameter has a weight 
International Journal of Computer Networks \& Communications (IJCNC) Vol.3, No.2, March 2011

associated with it. Using the weights the digression for network is computed as: $\Delta D_{i}=\sum_{j=1}^{P} W_{j} \Delta D_{i j}$.

\section{FRAMEWORK IMPLEMENTATION}

In this section we provide an example implementation of our framework. Figure 5 depicts this example. The handover decision algorithm resides in the mobile device in this particular example, but it is possible to have the handover decision algorithm in a network element in the case of a network controlled handover. In this example we utilize the IEEE 802.21 standard [4] known as Media Independent Handover (MIH). This is the standard for optimization of handovers between IEEE 802 systems and cellular systems.

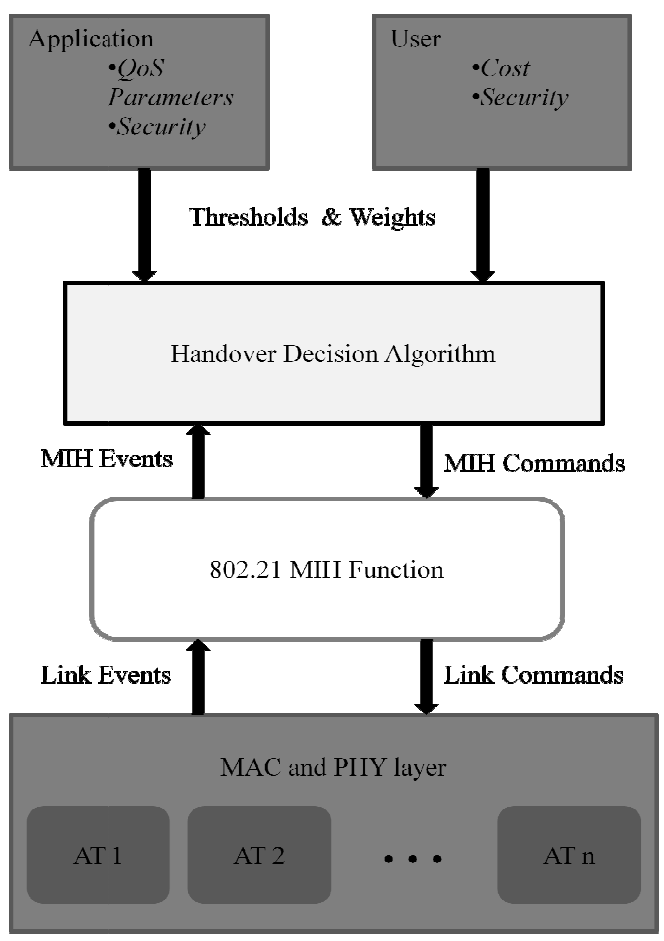

Figure 5 Framework Implementation Example

The entities providing the parameters for the handover decision are:

MAC and PHY layer: The PHY layer parameters used are signal-to-noise ratio (SNR), BER, and received signal strength (RSS). Only the mandatory thresholds are considered for the PHY layer. The MAC layer (link layer) parameters specify the parameters measured by the MAC layers, such as the peak rate, packet error rate, jitter, etc. The mandatory and preferred thresholds set for these parameters are dependent on the application used. Due to the different access networks supported, the MAC and PHY layer are specific to the access technology (AT) of the networks, such as 802.3 or radio access technologies such as $802.11,802.16$ or UMTS.

Application: The application entity provides the parameters that affect the performance of the application/service used by the mobile device. In this example we use the following application parameters:

QoS Requirements: The implementation can utilize the QoS parameters specified in the 802.21 standard: data rate, minimum packet transfer delay, maximum packet transfer delay, jitter, 
packet loss rate, and packet error rate [4]. An application requires all or a subset of these parameters. The application provides thresholds and weights for each of the parameters it specifies. We consider both mandatory and preferred thresholds for these parameters in this example.

User: The user provides a profile that includes the parameters of:

Cost: This requirement specifies the monetary cost that the user is ready to incur due to a connection.

Security: With this requirement the user specifies the authentication related requirements. For example, the authentication requirements can be given as different levels where level 0 indicates open authentication, level 1 indicates a shared key based authentication with no encryption, and level 2 may indicate shared key based authentication with encryption etc.

Now, we elaborate on the operation of the handover decision algorithm using this example framework. Figures $6 \mathrm{a}$ and $6 \mathrm{~b}$ show handover initiation due to the PHY layer mandatory thresholds not satisfied and due to the application QoS requirements not met.

The handover decision algorithm is notified that the PHY layer mandatory thresholds are not met through the event service provided by the 802.21 MIHF. A protocol that wishes to use the MIH event service sends the message MIH_Event_Subscribe.request [13] to the MIHF as shown in Figure 6a. In the case of the PHY layer mandatory thresholds, this is achieved by subscribing to the predictive event Link_Going_Down of the current link. This event is triggered by the current MAC and PHY layer, whenever the mandatory thresholds of PHY layer parameters of this link are not met.

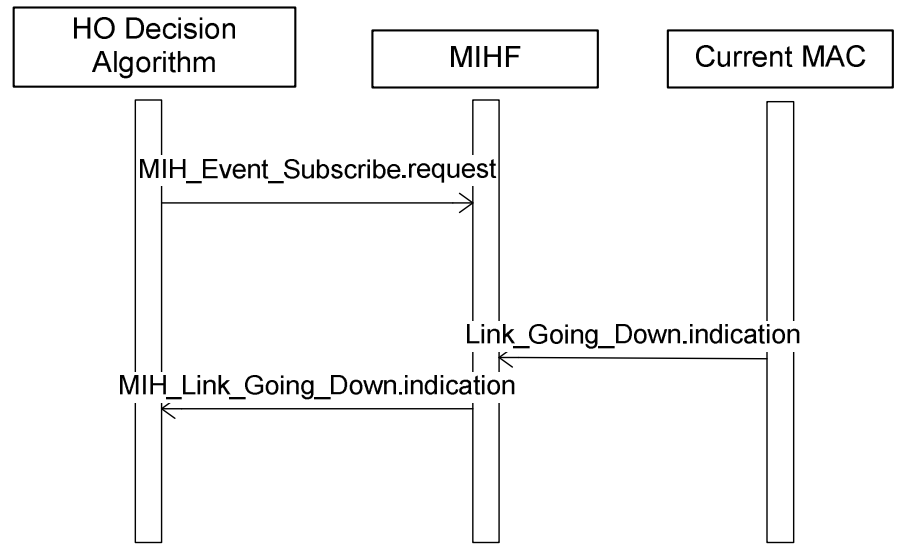

Figure 6a. Notification of PHY layer Performance

In order to get notified whenever the mandatory thresholds of the QoS requirements are not satisfied, we utilize the MIH_Link_Configure_Thresholds command [4] as shown in figure 6b. The 802.21 standard provides the mapping of the specified QoS parameters to AT specific parameters. When the MIHF receives the MIH_Link_Configure_Thresholds request, it maps the QoS parameter thresholds to the current link layer parameters, and sends the command Link_Configure_Thresholds to the current link layer. This results in the link layer notifying the MIHF using Link_Parameters_Report event whenever the specified thresholds are not met. 


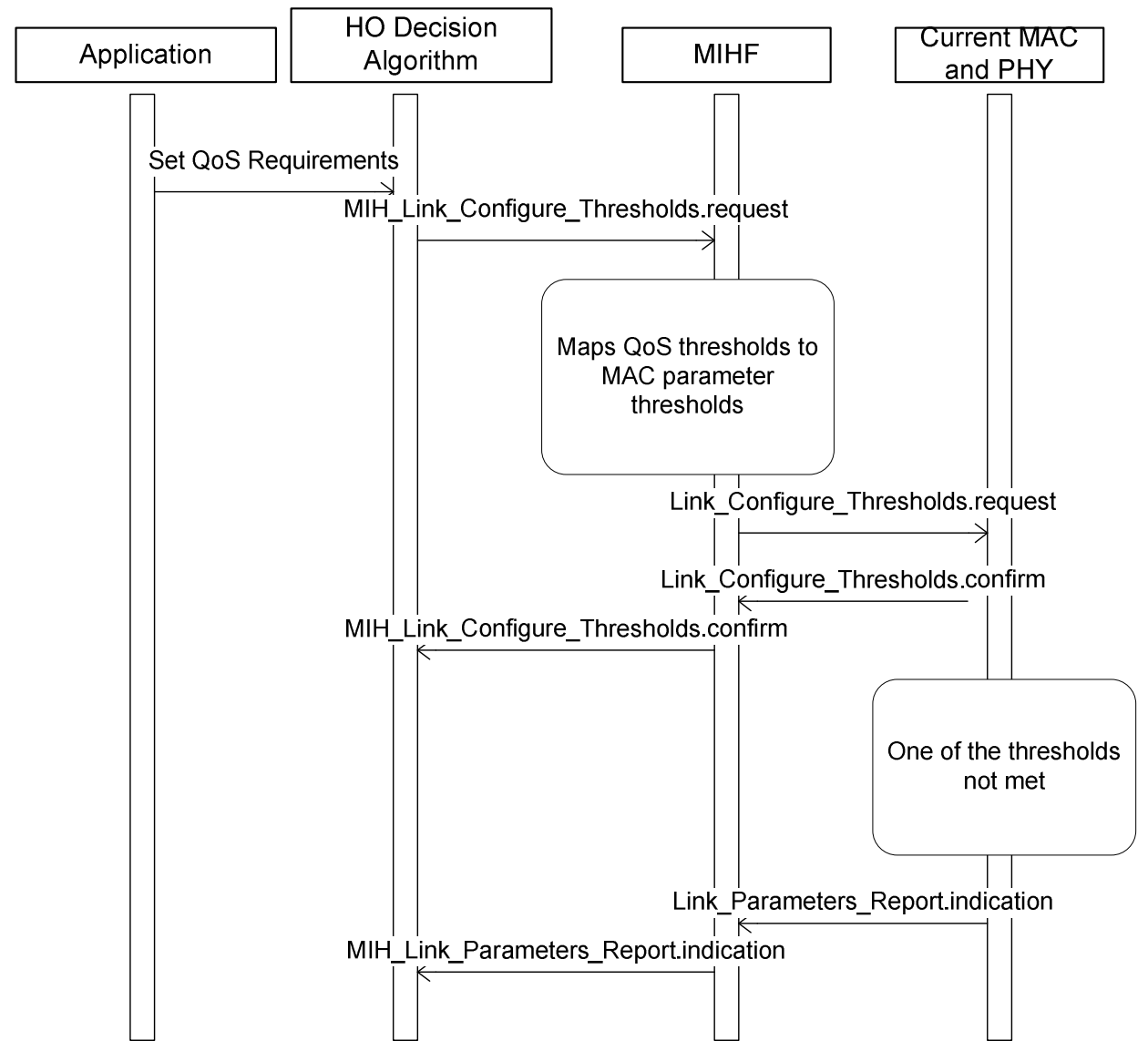

Figure 6b Notification of Application QoS Deterioration

In Figure 7, we show when the network selection algorithm is triggered and how it acquires the network offered values. When the mobile node (MN) first connects to any network, it acquires information about neighbor networks through the MIH_Get_Information.request message of the 802.21 media independent information service (MIIS). The 802.21 standard specifies a MIIS server that serves a group of neighboring networks and has static knowledge of the network parameters such as the network identification, cost of service, and security offered. The MIIS server responds to the information request from the MIHF of the MN with this information. This provides the values offered for cost and security parameters.

As described in section 5.2, one of the conditions for handover initiation is that a neighboring network is detected. This is achieved in the example through the MIHF event Link_detected. The other condition to initiate handover is whenever the mandatory thresholds of any parameters are not satisfied. As described above the events Link_going_down and Link_Parameters_Report notify that the mandatory thresholds of the PHY layer parameters and the application QoS parameters respectively are not met.

When handover is initiated, the handover decision algorithm acquires the network offered values of all the available links for the specified parameters through the request MIH_HO_Candidate_Query to the MIHF. The MIHF in turn requests the information from the current network which in turn requests the information from the given neighboring networks. With this information, the network selection algorithm has all the required values to calculate the digression of all the neighboring networks and select a network to handover to. 
International Journal of Computer Networks \& Communications (IJCNC) Vol.3, No.2, March 2011



Figure 7 Network Selection Procedure

\section{SimULATION AND RESULTS}

We simulated our handover decision algorithm and the SAW algorithm as used by Zhu and McNair [7] and the GRA algorithm as given by Song and Jamalipour [9].

\subsection{Simulation Model}

For our simulations, we used a discrete event simulator developed by us using the Microsoft Visual Studio .Net C++ to simulate mobility and handovers. We have used a network area of 2000 square meters populated by three different access networks (ANs) as shown in Figure 8.We use shadow fading with $6 \mathrm{~dB}$ standard deviation to calculate the RSS at the mobile node. The specifications of the three ANs for this calculation are given in Table 1.

Table 1. Network Specifications for RSS Calculation

\begin{tabular}{|l|l|l|l|}
\hline & AN 1 & AN 2 & AN 3 \\
\hline Frequency (MHz) & 2600 & 2300 & 1900 \\
\hline Transmit Power (dBm) & 50 & 40 & 33 \\
\hline Coverage Radius (meters) & 1000 & 500 & 250 \\
\hline
\end{tabular}




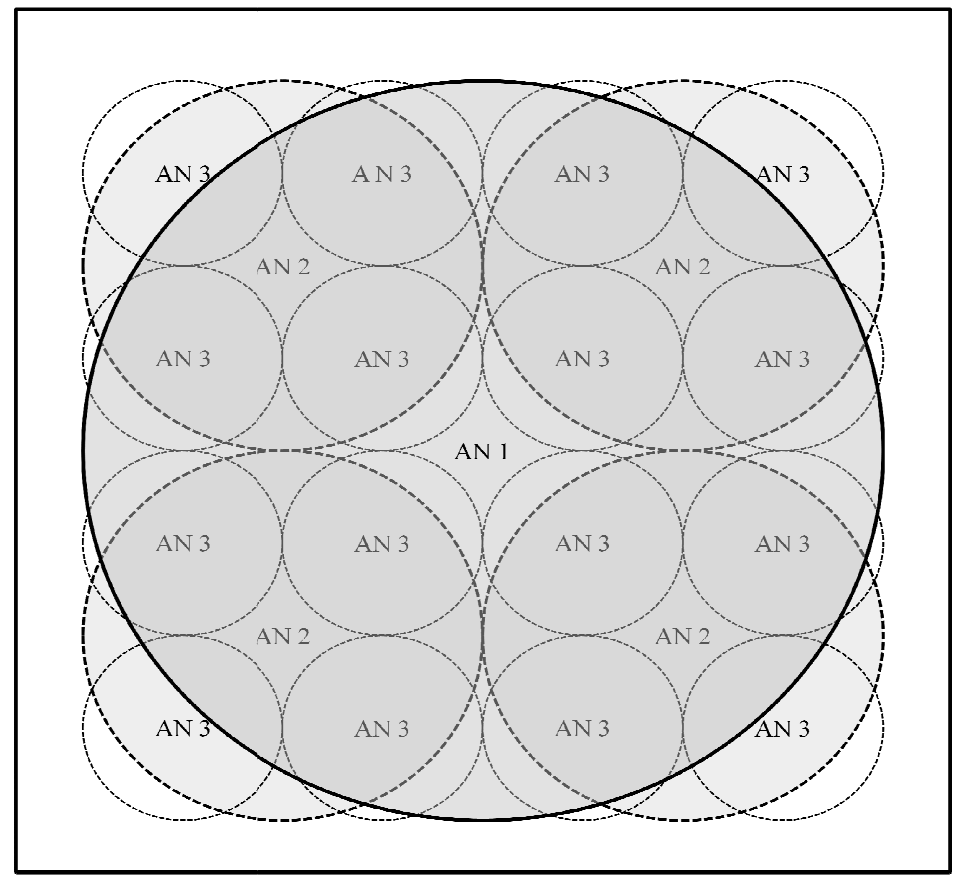

Figure 8 Simulation Network Model

The parameters used for handover decision are the received signal strength (RSS), data rate offered, and the cost of AN. Only the mandatory threshold is considered for the RSS parameter; for the data rate and cost parameters both mandatory and preferred thresholds are considered. While the RSS value is dynamic and changes with the location of the mobile node, data rate and cost are static parameters. We have used the random waypoint mobility model [11], where initially the mobile node is at a random position within the simulation area. Subsequently, the $\mathrm{MN}$ randomly chooses a new location and moves to this location with a speed of $5 \mathrm{~m} / \mathrm{s}$.

The simulation is done for two scenarios:

i. In the first scenario there is a large discrepancy between the values offered by the three access networks and only one network satisfies the preferred threshold for the cost parameter. Table 3 shows the values offered by the networks. We can see that in the first scenario the difference between the data rate offered by the networks AN 1 and AN 3 is vastly higher than the data rate of $8 \mathrm{Mbps}$ offered by AN 2. Only AN 2 satisfies the preferred threshold of 0.3 for cost in this scenario.

ii. In the second scenario the discrepancy between the values offered is less and two of the three available networks satisfy the preferred threshold. As can be seen from Table 3, in this scenario the data rates offered by the three networks differ at most by $150 \mathrm{Mbps}$ and access networks 1 and 2 both satisfy the preferred thresholds for both data rate and cost.

We provide the details of the requirements for the parameters and the values offered by the networks for each of the scenarios in Tables 2 and 3. Cost is given as a normalized value using the highest cost charged by a network. For example if the highest cost charged is by a network is $\$ 100$, then the cost value for AN 1 is 0.6 if this network charges $\$ 60$. 
International Journal of Computer Networks \& Communications (IJCNC) Vol.3, No.2, March 2011

Table 2. Parameter Requirements.

\begin{tabular}{|l|l|l|l|l|}
\hline \multirow{2}{*}{} & \multicolumn{2}{|l|}{ Mandatory Threshold } & \multicolumn{2}{l|}{ Preferred Threshold } \\
\cline { 2 - 5 } & Data rate & Cost & Data rate & Cost \\
\hline Scenario 1 & $4 \mathrm{Mbps}$ & 0.6 & $8 \mathrm{Mbps}$ & 0.3 \\
\hline Scenario 2 & $25 \mathrm{Mbps}$ & 0.6 & $50 \mathrm{Mbps}$ & 3 \\
\hline
\end{tabular}

Table 3. Parameter Values

\begin{tabular}{|l|l|l|l|l|l|l|}
\hline & \multicolumn{2}{|l|}{ Data Rate } & \multicolumn{2}{l|}{ Cost } \\
\cline { 2 - 7 } & AN 1 & AN 2 & AN 3 & AN 1 & AN 2 & AN 3 \\
\hline Scenario 1 & $100 \mathrm{Mbps}$ & $8 \mathrm{Mbps}$ & $200 \mathrm{Mbps}$ & 0.6 & 0.3 & 0.4 \\
\hline Scenario 2 & $100 \mathrm{Mbps}$ & $50 \mathrm{Mbps}$ & $200 \mathrm{Mbps}$ & 0.6 & 0.3 & 0.3 \\
\hline
\end{tabular}

\subsection{Results and Analysis}

We present the results for both the scenarios in terms of the number of handovers occurred and the percentage of handovers that satisfies the preferred thresholds. In the results given below we are only recording those handovers that occurred when more than one AN was available for selection, and when AN 2 was one of those networks. This is done in order to evaluate the behavior of the algorithms at times when a network satisfying preferred thresholds is available. Figure 9 compares the total number of handovers and Figure 10 gives the percentage of times the preferred thresholds were met after the handovers.

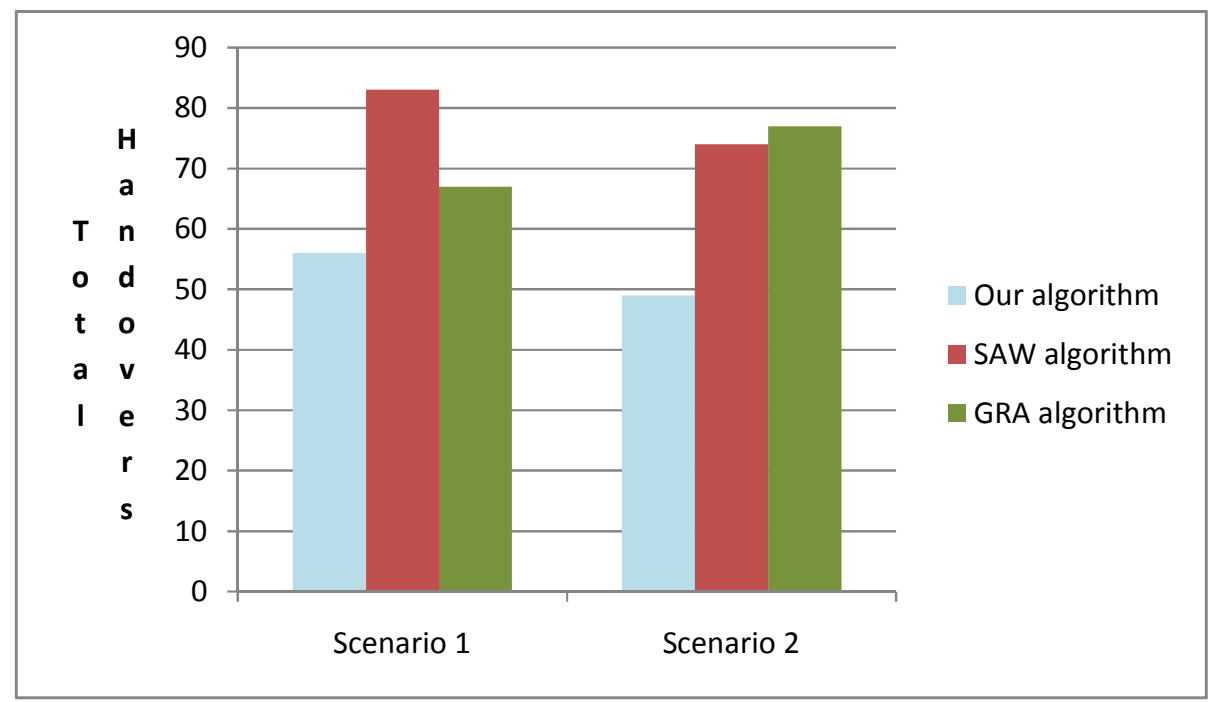

Figure 9 Simulation Results: Total Handovers 
The results show that by considering the preferred thresholds, our algorithm avoids unnecessary handovers. In Figure 9, we observe this for both the scenarios, even though in the second scenario both networks AN 1 and AN 2 satisfy the preferred thresholds. This is due to the fact that the MN executes handover only if the current network does not satisfy the preferred thresholds. Hence by utilizing preferred thresholds for parameters we reduce the handovers by more than $25 \%$. Handover execution not only requires resources of the user device and networks, but also the bandwidth which is limited for wireless access networks. Hence, by reducing the unnecessary handovers through our handover decision algorithm, we have achieved resource efficiency.

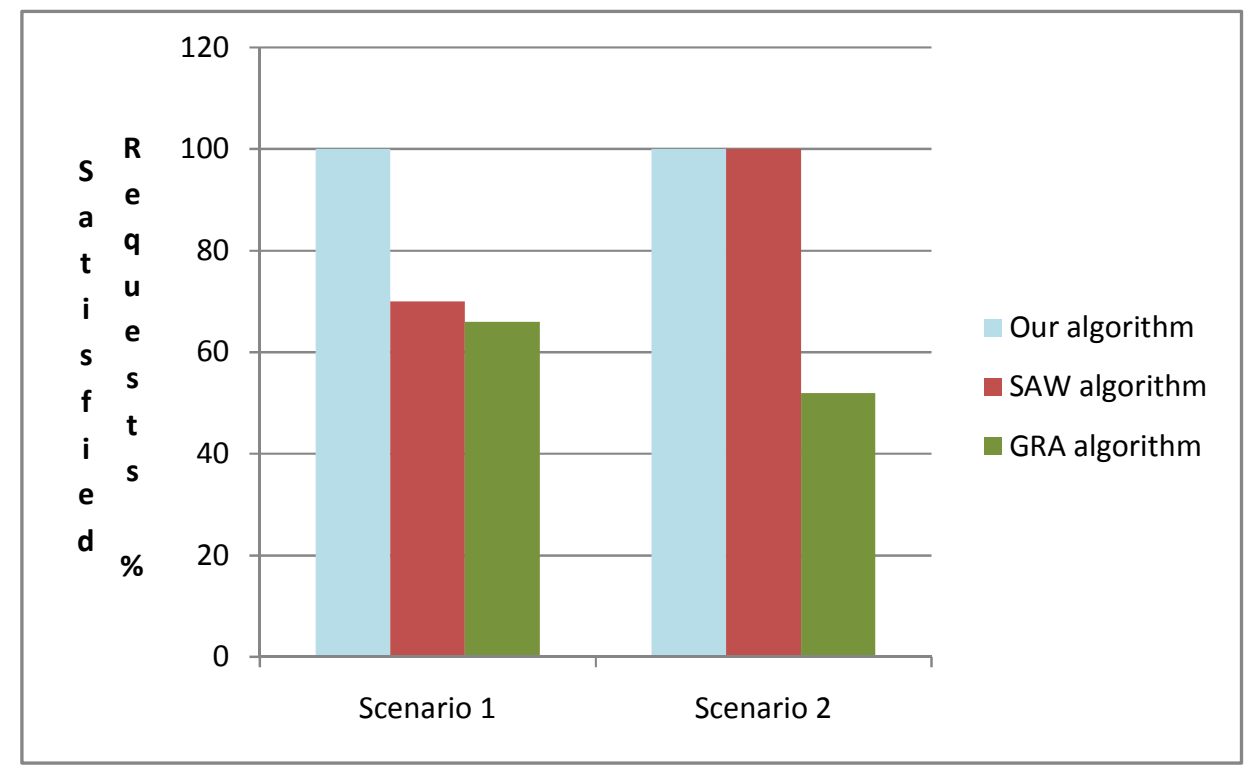

Figure 10. Percentage of Preferred Requests Satisfied

In the first scenario, only AN 1 can satisfy the preferred thresholds of all parameters. The preferred thresholds are satisfied during hundred percent of the handovers when our algorithm is used as shown in Figure 10. This is due to the fact that by using digression our algorithm always chooses the network that most satisfies the preferred thresholds. In the second scenario where the two networks AN2 and AN3 satisfy the preferred thresholds, SAW based algorithm also satisfies the preferred thresholds. Availability of different access networks offering values that differ largely is a definite possibility in NGNs. We have shown that in such scenarios our algorithm satisfies the parameter requirements whenever possible.

\section{Conclusions}

The goal of the next generation networks is to provide services to users utilizing a packet based core network which is able to integrate a variety of transport technologies, wired and wireless. The ITU-T standardization body has published requirements for the NGNs. In this paper we have considered the handover decision problem, which is complex due to the availability of different access networks. Next generation networks not only offer the choice of a variety of access networks but also a myriad of applications to users. A key feature of the applications designed for NGNs is their adaptability to the diverse networks offered. The overall user experience depends not only on the performance of the applications, but also on the cost benefits and the device performance. We have presented a generic framework for handover decision in NGNs using the concept of preferred thresholds for the requirements of parameters that affect the handover. Through examples we showed that our framework can be utilized by any existing handover decision algorithm for horizontal or vertical handover. 
International Journal of Computer Networks \& Communications (IJCNC) Vol.3, No.2, March 2011

We have presented a handover decision algorithm that utilizes the concept of preferred thresholds and hence represents the adaptive nature of the NGN services. Through simulations we have compared our algorithm with the vertical handover decision algorithms based on SAW [25] and GRA [27]. We observe that our algorithm is able to satisfy the user requirements and, in scenarios where there is a considerable discrepancy in the values offered by networks for the different parameters, only our algorithm picks the network that most satisfies the user preference during all handovers. The results showed that in all scenarios we reduce handovers by more than $25 \%$, thus improving resource efficiency.

\section{REFERENCES}

[1] ITU-T Recommendation Q.1706 "Next Generation Networks: Generalized Mobility" International Telecommunication Union, 2006

[2] F. Cacace and L. Vollero, "Managing Mobility and Adaptation in Upcoming 802.21 Enabled Devices," Proc. 4th Int'l. Wksp. Wireless Mobile Apps. and Services on WLAN Hotspots, 2006

[3] G. Lampropoulos, A. Salkintzis, and N. Passas, "Media Independent Handover for Seamless Service Provision in Heterogeneous Networks," IEEE Commun. Mag., vol.46, no. 1, Jan. 2008.

[4] IEEE Std. 802.21-2008. "IEEE standard for Local and metropolitan area networks- Part 21: Media Independent Handover"

[5] 3gPP TS23.060 v 6.9.0, "General packet radio service". 3GPP specifications

[6] Y. Choi and S. Choi, "Service charge and energy-aware vertical handoff in integrated IEEE 802.16e/802.11 networks,” Proc. IEEE INFOCOM, 2007, pp. 589-597.

[7] Fang, Z., McNair, J.: “Multiservice Vertical Handoff Decision Algorithms.” EURASIP Journal on Wireless Communications and Networking 2006(2), 52-64 (2006)

[8] Rami T, O. Salazar, Guy P. "Vertical Handoff Decision Scheme Using MADM for Wireless Networks". In IEEE WCNC 2008

[9] Q. Song and A. Jamalipour, "A network selection mechanism for next generation networks," in Proc. IEEE ICC, Seoul, Korea, May 2005, pp. 1418-1422.

[10] G. Pollini, "Trends in handover design," IEEE Commun. Mag., vol. 34, no. 3, pp. 82-90, Mar. 1996.

[11] J. Broch, D. A. Maltz, D. B. Johnson, Y. Hu, and J. G. Jetcheva. A Performance Comparison of Multi-Hop Wireless Ad Hoc Network Routing Protocols. In MobiCom'98, Proceedings of the Fourth Annual ACM/IEEE International Conference on Mobile Computing and Networking , pp 85-97.

[12] Pen-ho Chang and Tsan-Pin Wang (2009) "Design and Implementation of an Integrated RFID and VOIP System for Supporting Personal Mobility" International Journal of Computer Networks \& Communications (IJCNC) October 2009 vol 1, no. 3.

[13] Yee YC, et al. (2007) "SIP-based proactive and adaptive mobility management framework for heterogeneous networks", Journal of Network and Computer Applications.

[14] Dutta et al. 2005) "Seamless Handover across Heterogeneous Networks - An IEEE 802.21 Centric Approach" Proceedings of the international wireless submit-wireless personal multimedia communications (WPMC) 2005.

[15] Sricharan, M.S.; Vaidehi, V.; Arun, P.P. (2006) "An activity based mobility prediction strategy for next generation wireless networks" Proceedings of IFIP International Conference on Wireless and Optical Communications Networks, 2006. 
International Journal of Computer Networks \& Communications (IJCNC) Vol.3, No.2, March 2011

Authors

Shanthy Menezes received the M.S. and Ph. D degrees in Computer Science from the University of Texas at Dallas in 2000 and 2010 respectively. Her research interests include wireless networks, mobility management in next generation heterogeneous networks, and network simulation.

S. Venkatesan received the M.S. and Ph.D. degrees from the University of Pittsburgh in 1985 and 1989, respectively and has been a faculty member in the Computer Science Department at the University of Texas at Dallas since then. His interests are in telecommunication networks, various issues in the design and operation of wireless networks, distributed algorithms, fault-tolerance and network forensics and security. His work has been funded by the federal, state and industrial sponsors in the past. 